Proyecciones

Vol. 20, No 2, pp. 193-204, August 2001.

Universidad Católica del Norte

Antofagasta - Chile

\title{
ATTRACTORS POINTS IN THE AUTOSUBSTITUTION *
}

\author{
EDUARDO MONTENEGRO \\ Universidad Técnica Federico Santa María, Chile \\ and \\ EDUARDO CABRERA \\ Universidad de Playa Ancha, Valparaíso - Chile
}

\begin{abstract}
Recently an operation of graphs called substitution has been incorporated. In an informal way, the substitution consists in the replacement of a vertex for a graph. This new graph is characterized through a function (of substitution) that it could be self definable. The substitution of each vertex of a graph $G$, through injectives functions of substitution, by the same $G$ graph will be called autosubstitution and denoted by $G(G)$. If $X$ represents the class of all the simple and finite graphs and $w$ is an application of $X$ in $X$, defined by $w(G)=$ $G(G)$, then it is interest in studying the dynamic properties of $w$ and the construction of some algorithms that they permit the generating of fractal images. In function of the above-mentioned it is proposed to analyze the autosubstitution for graphs simple and finite. Framed in the area of the Graph Dynamics, inside the area of the Graph Theory, the present work will use, preferably, simple and finite graph.
\end{abstract}

Key words : Graph, substitution of graph, discrete dynamical systems.

AMS subject classifications : 05C25;05C35.

${ }^{*}$ Work financed by the General Address of Investigation of the UPLACED through the Project CNEI 060001. 


\section{Introduction.}

The graph to considered will be in general simple and finite, graphs with a nonempty set of edges. For a graph $G, V(G)$ denote the set of vertices and $E(G)$ denote the set of edges. The cardinality of $V(G)$ is called order of $G$ and the cardinality of $E(G)$ is called size of $G$. A $(p, q)$ graph has order $p$ and size $q$. Two vertices $u$ and $v$ are called neighbors if $\{u, v\}$ is an edge of $G$. For any vertex $v$ of $G$, denote by $\mathrm{Nv}$ the set neighbors of $\mathrm{v}$. To simplify the notation, and edge $\{\mathrm{x}, \mathrm{y}\}$ is written as xy (or yx). Other concepts used in this work and not defined explicitly can be found in the references [1], [2], [3], [5], [9], [12], [13].

\section{Preliminaries}

. Some essential concepts of this work are the following :

\subsection{The Substitution [10], [11]}

It assumes that $\mathrm{G}$ and $\mathrm{K}$ and two disjointed graphs by vertices. For a vertex $\mathrm{v}$ in $\mathrm{V}(\mathrm{G})$ and a function $\mathrm{s}: \mathrm{N}_{v} \rightarrow \mathrm{V}(\mathrm{K})$ it will be defined the substitution of the vertice $\mathrm{v}$ by the graph $\mathrm{K}$, as the graph $\mathrm{M}$, denoted by $\mathrm{G}(\mathrm{v}, \mathrm{s}) \mathrm{K}$, such that:

(1) $\mathrm{V}(\mathrm{M})=(\mathrm{V}(\mathrm{G}) \cup \mathrm{V}(\mathrm{K}))-\{\mathrm{v}\}$ and

(2) $\mathrm{E}(\mathrm{M})=\left(\mathrm{E}(\mathrm{G})-\left\{\mathrm{vx} / \mathrm{x} \in \mathrm{N}_{v}\right\}\right) \cup\left\{\mathrm{xs}(\mathrm{x}) / \mathrm{x} \in \mathrm{N}_{v}\right\}$.

The vertex $\mathrm{v}$ is said to be the substitution vertex by $\mathrm{K}$ in $\mathrm{G}$ under the function $\mathrm{s}$ and this function is called substitution function. Figure 1 shows an example of substitution. 


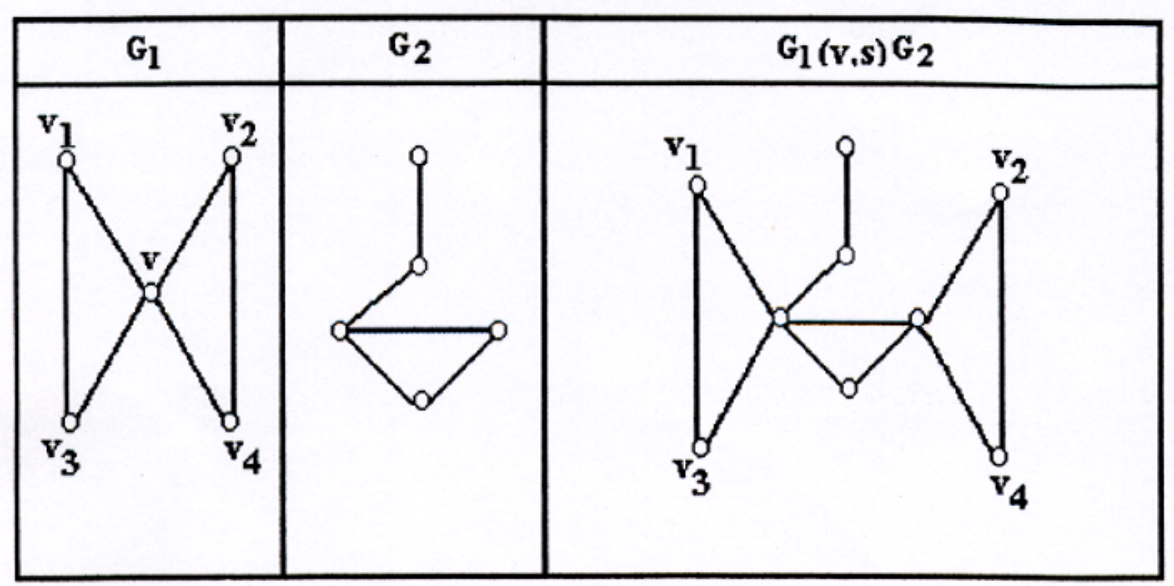

FIGURE 1

Now let $\mathrm{v}_{1}, \ldots, \mathrm{v}_{n}$ be the vertices of a graph $\mathrm{G}$ and $\mathrm{H}_{1}, \ldots, \mathrm{H}_{n}$ a sequence of graphs with no common vertices among themselves or with G. By $\mathrm{M}_{k}=\mathrm{M}_{k-1}\left(\mathrm{v}_{k}, \mathrm{~s}_{k}\right) \mathrm{H}_{k}$ it will be denoted the graph which is obtained by substitution of vertices of $\mathrm{G}$ by graphs $\mathrm{H}_{i}, 1 \leq i \leq \mathrm{k}$, where $\mathrm{M}_{0}=\mathrm{G}$. In other words, $\mathrm{M}_{1}$ denotes a graph obtained by substitution of only one vertex of $\mathrm{G}, \mathrm{M}_{2}$ denotes a graph obtained by substitution of only one vertex of $\mathrm{M}_{1}$, and so on. Note that every substitutes vertex must belong to $\mathrm{V}(\mathrm{G})$. Figure 2 shows an example of $\mathrm{M}_{6}$. 


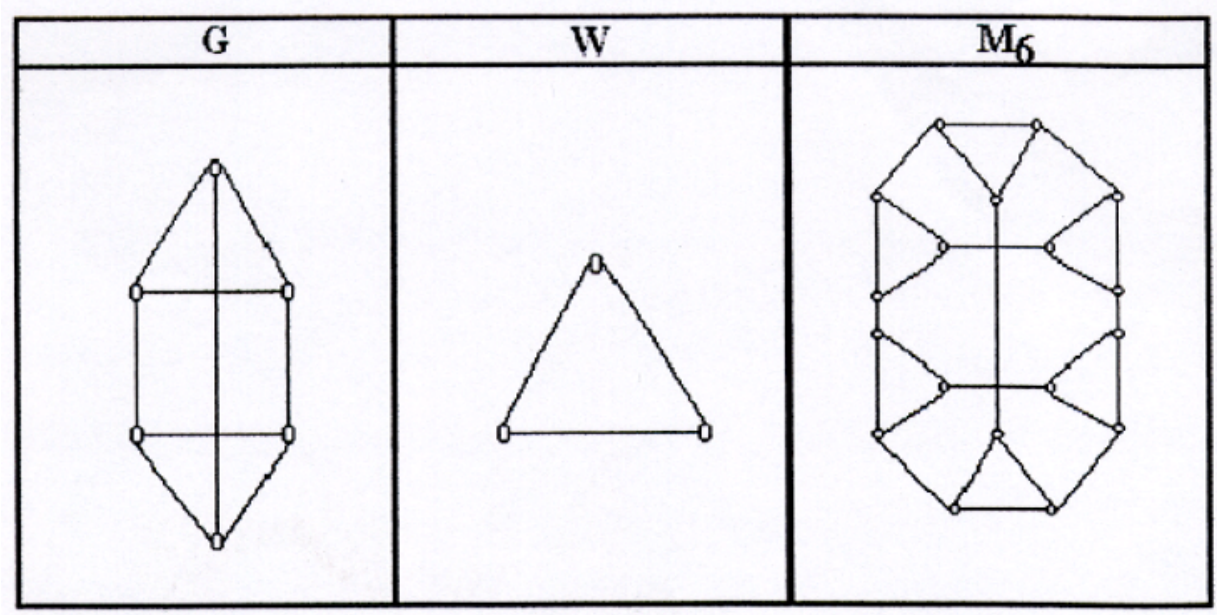

FIGURE 2

It can be said that an edge of the substitution $\mathrm{M}_{p}$ is an edge internal [10] if it is denoted by $\mathrm{s}_{i}(\mathrm{x}) \mathrm{s}_{i}(\mathrm{y})$. The edge in $\mathrm{M}_{p}$ that is not edge internal will be nominated edge external [9]. Let $\mathrm{G}$ be a graph without isolated vertices. If each vertex $\mathrm{v}$ of $\mathrm{G}$ is substituted by a complete graph with $\operatorname{val}(\mathrm{v})$ vertices, through an injective function, then it will be said that the graph $G$ has been expanded [9]. When each vertex of a given $\mathrm{G}$ graph is substituted for a copy [3] of $\mathrm{G}$ through injectives functions of substitution is obtained a special type of substitution which will be called autosubstitution and denoted by $G(G)$. If $G$ is a cycle then $\mathrm{G}(\mathrm{G})$ will be called autocycle. Figure 3 shows an example of $\mathrm{G}(\mathrm{G})$ for $\mathrm{G}=\mathrm{C}_{4}$. 


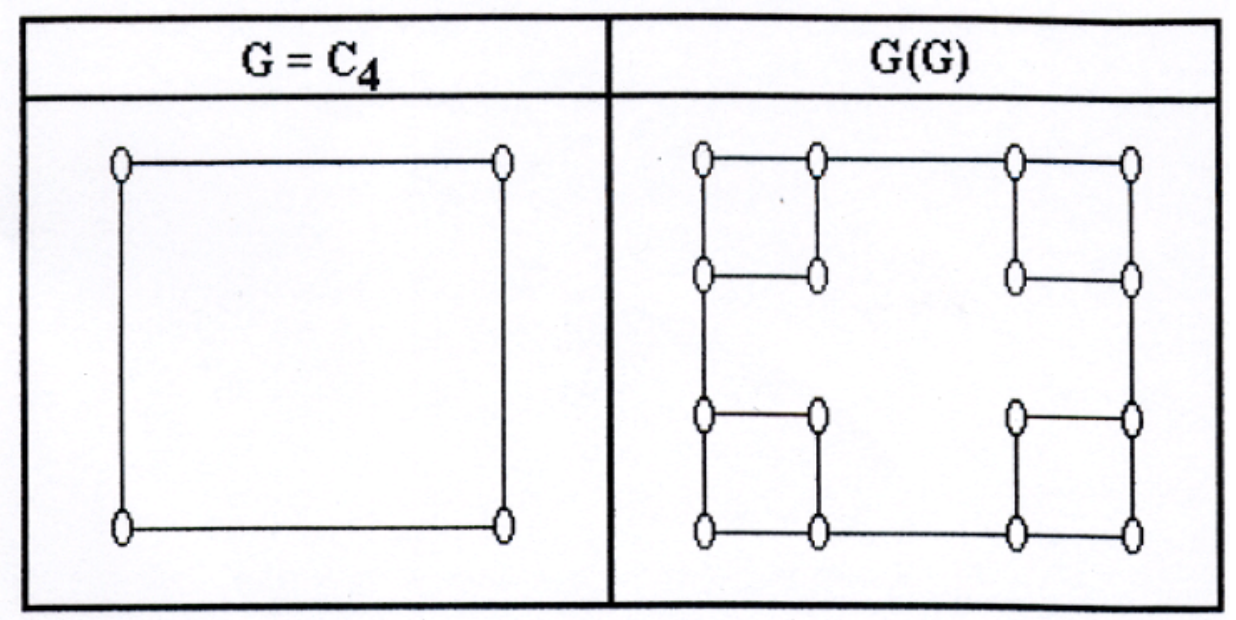

FIGURE 3

\subsection{Realizable graph [2], [3]:}

A (p,q) graph $\mathrm{G}$ is said to be realizable on $\mathbf{R}^{\mathbf{3}}$ if it is possible to distinguish a collection of $\mathrm{p}$ distinct point of $\mathbf{R}^{\mathbf{3}}$ that correspond to the vertices of $\mathrm{G}$ and a collection of q curves, pairwise disjoint except possibly for endpoint, on $\mathbf{R}^{\mathbf{3}}$ that correspond to the edges of $\mathrm{G}$ such that if a curve $\lambda$ corresponds to the edge $\mathrm{e}=\mathrm{uv}$, then only the endpoints of $\lambda$ correspond to vertices of $G$, namely $u$ and $v$.

\subsection{Discrete dynamical systems [6], [7], [12].}

A discrete dynamical system is any set $\mathrm{X}$ together with a mapping $f: \mathrm{X} \rightarrow \mathrm{X}$. In this work $\mathrm{X}$ is always a set of graph. In the literature [12], $\mathrm{X}$ must be some topological space such that $f$ is continuous. An orbit of $f$ in $G$ is any set of the form $\left\{\mathrm{G}, f(\mathrm{G}), \ldots, f^{n}(\mathrm{G}), \ldots\right\}$. A graph $\mathrm{G}$ is an attractor point of $f$ if there is some natural $n>0$ with $\mathrm{G}=f^{n}(\mathrm{G})$ and $\mathrm{G} \neq f^{t}(\mathrm{G})$ if $t<n$. 


\section{Attractors Points}

- The class of all the simple and finite graphs will be written down by $\mathrm{X}$ [6] and an application $\mathrm{X}$ in $\mathrm{X}$ that takes a $\mathrm{G}$ graph in $\mathrm{G}(\mathrm{G})$ will be denoted by $w$. It is of noticing that each element of the orbit of $w$ is obtained by substitution of each one of its vertices by $\mathrm{G}$.

The realization of the graph $G$ in $\mathbf{R}^{\mathbf{3}}$ will be denoted by $\mathrm{C}(\mathrm{G})$.

If $h: V(\mathrm{G}) \rightarrow \mathbf{R}^{\mathbf{3}}$ is a injective function defined by $h(v)=\tilde{v}$ and if $\mathrm{V}(\mathrm{G})=\left\{v_{1}, \ldots, v_{n}\right\}$, then $\mathrm{C}(\mathrm{G})=\bigcup_{v_{i} v_{j} \in E(G)} \tilde{v}_{i} \tilde{v}_{j}$, where $\tilde{v}_{i} \tilde{v}_{j}=$ $\left\{\tilde{v}_{i}-\lambda\left(\tilde{v}_{j}-\tilde{v}_{i}\right) / \lambda \in[0,1]\right\}$. If $\mathrm{E}(\mathrm{G})=\Phi$ and $V(\mathrm{G})=\left\{v_{1}, \ldots, v_{n}\right\}$, then $\mathrm{C}(\mathrm{G})=\left\{\tilde{v}_{1}, \ldots, \tilde{v}_{n}\right\}$.

Lemma 3.1: $\mathrm{C}(\mathrm{G})$ is compact in $\mathbf{R}^{\mathbf{3}}$.

\section{Proof :}

(i) $\mathrm{C}(\mathrm{G})$ is bounded.

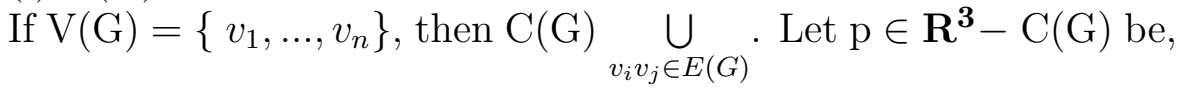
then $\mathrm{d}\left(\mathrm{p}, \tilde{v}_{i}\right)>0, i \in\{1, \ldots, n\}$, where d denotes the usual distance in $\mathbf{R}^{3}$. It is defined $r=2 v_{i} \in V(G) \max \mathrm{d}\left(\mathrm{p}, \tilde{v}_{i}\right)$ and consequently $\mathrm{C}(\mathrm{G})$ $\subset V_{r}(p)$.

(ii) $\mathrm{C}(\mathrm{G})$ is closed.

Let $\mathrm{p} \in \mathbf{R}^{\mathbf{3}}-\mathrm{C}(\mathrm{G})$ be, then it is defined $r \in \mathbf{R}^{+}$by $\mathrm{d}(\mathrm{p}, \mathrm{C}(\mathrm{G}))$ and consequently $V_{r}(p) \subset \mathbf{R}^{\mathbf{3}}-\mathrm{C}(\mathrm{G})$. Therefore $\mathrm{C}(\mathrm{G})$ is closed $\bullet$

Let $A_{\lambda}$ be the set defined by $\left\{y \in \mathbf{R}^{3} / \exists x \in A, d(x, y)<\lambda\right\}$ where $A \subset \mathbf{R}^{\mathbf{3}}, \lambda \in \mathbf{R}^{+}$. Is obvious that $A_{\lambda}=\bigcup_{a \in A}$, where $V_{\lambda}(a)=\{$ $\left.x \in \mathbf{R}^{\mathbf{3}} / \mathbf{d}(\mathbf{x}, \mathbf{a})<\lambda\right\}$.

Let $A \subset \mathbf{R}^{\mathbf{3}}$ be a bounded set. The diameter of $A$, denoted by $\operatorname{diam}(A)$, is the real number $\max _{x, y \in A}$, i.e., $\operatorname{diam}(A)=x, y \in A \max$ $d(x, y)$.

Moreover, if $A, B, C \subset \mathbf{R}^{\mathbf{3}}$, then $. d(A, B) \leq d(A, C)+d(C, B)$.

From now on, by $\mathrm{X}$ it will be denoted the class of all the simple and finite graphs. 
In the following proposition it will be constructed a distance in X.

Proposition 3.2: The function $\delta: X \times X \rightarrow \mathbf{R}$ defined by $\delta\left(G_{1}, G_{2}\right)=\inf \left\{\lambda \in \mathbf{R}^{+} / \mathbf{C}\left(\mathbf{G}_{\mathbf{1}}\right) \subset\left(\mathbf{C}\left(\mathbf{G}_{\mathbf{2}}\right)\right)_{\lambda} \wedge \mathbf{C}\left(\mathbf{G}_{\mathbf{2}}\right) \subset\left(\mathbf{C}\left(\mathbf{G}_{\mathbf{1}}\right)\right)_{\lambda}\right\}$, is a distance on $X$.

Proof : If $\mathrm{G}, \mathrm{G}_{1}, \mathrm{G}_{2}, \mathrm{G}_{3} \in X$, then

(i) Since $\delta(\mathrm{G}, \mathrm{G})=\inf \left\{\lambda \in \mathbf{R}^{+} / \mathbf{C}(\mathbf{G}) \subset(\mathbf{C}(\mathbf{G}))_{\lambda}\right\}$ and $\mathrm{C}(\mathrm{G})$ is compact in $\mathbf{R}^{\mathbf{3}}$, then $\delta(\mathrm{G}, \mathrm{G})=0$.

(ii) Suppose $\delta\left(G_{1}, G_{2}\right)>0$.

Since $\inf \left\{\lambda \in \mathbf{R}^{+} / \mathbf{C}\left(\mathbf{G}_{\mathbf{1}}\right) \subset\left(\mathbf{C}\left(\mathbf{G}_{\mathbf{2}}\right)\right)_{\lambda} \wedge \mathbf{C}\left(\mathbf{G}_{\mathbf{2}}\right) \subset\left(\mathbf{C}\left(\mathbf{G}_{\mathbf{1}}\right)\right)_{\lambda}\right\}>$ $\mathbf{0}$, then $G_{1} \neq G_{2}$.

Suppose $. G_{1} \neq G_{2}$

Since $\delta\left(G_{1}, G_{2}\right)=\inf \left\{\lambda \in \mathbf{R}^{+} / \mathbf{C}\left(\mathbf{G}_{\mathbf{1}}\right) \subset\left(\mathbf{C}\left(\mathbf{G}_{\mathbf{2}}\right)\right)_{\lambda} \wedge \mathbf{C}\left(\mathbf{G}_{\mathbf{2}}\right) \subset\right.$ $\left.\left(\mathbf{C}\left(\mathbf{G}_{1}\right)\right)_{\lambda}\right\}$, and $C\left(G_{1}\right) \subset\left(C\left(G_{1}\right)\right)_{\lambda}, C\left(G_{2}\right) \subset\left(C\left(G_{2}\right)\right)_{\lambda}$, then $\left(C\left(G_{1}\right) \cup C\left(G_{2}\right)\right) \subset\left(C\left(G_{1}\right)\right)_{\lambda} \wedge\left(C\left(G_{1}\right) \cup C\left(G_{2}\right)\right) \subset\left(C\left(G_{2}\right)\right)_{\lambda} \Rightarrow$ $\Rightarrow\left(C\left(G_{1}\right) \cup C\left(G_{2}\right)\right) \subset\left(\left(C\left(G_{1}\right)\right)_{\lambda} \cap\left(C\left(G_{2}\right)\right)_{\lambda}\right) \Rightarrow$ $\Rightarrow \inf \left\{\lambda \in \mathbf{R}^{+} / \mathbf{C}\left(\mathbf{G}_{\mathbf{1}}\right) \subset\left(\mathbf{C}\left(\mathbf{G}_{\mathbf{2}}\right)\right)_{\lambda} \wedge \mathbf{C}\left(\mathbf{G}_{\mathbf{2}}\right) \subset\left(\mathbf{C}\left(\mathbf{G}_{\mathbf{1}}\right)\right)_{\lambda}\right\}>\mathbf{0} \Rightarrow$

(iii) By definition, $\delta\left(G_{1}, G_{2}\right)=\delta\left(G_{2}, G_{1}\right)$.

(iv) $\delta\left(G_{1}, G_{2}\right) \leq \delta\left(G_{1}, G_{3}\right)+\delta\left(G_{3}, G_{2}\right)$. $\delta\left(G_{1}, G_{2}\right) \leq \delta\left(G_{1}, G_{2}\right)+\operatorname{diam}\left(C\left(G_{3}\right)\right) \leq \operatorname{diam}\left(C\left(G_{3}\right)\right)+\operatorname{diam}\left(C\left(G_{1}\right)\right)+$ $+d\left(C\left(G_{1}\right), C\left(G_{2}\right)\right)+\operatorname{diam}\left(C\left(G_{2}\right)\right) \leq \operatorname{diam}\left(C\left(G_{3}\right)\right)+\operatorname{diam}\left(C\left(G_{2}\right)\right)+$ $+d\left(C\left(G_{1}\right), C\left(G_{3}\right)\right)+d\left(C\left(G_{3}\right), C\left(G_{2}\right)\right)+\operatorname{diam}\left(C\left(G_{3}\right)\right)+\operatorname{diam}\left(C\left(G_{1}\right)\right) \leq$ $\leq \delta\left(G_{1}, G_{3}\right)+\delta\left(G_{3}, G_{2}\right) \bullet$ 
Any important concepts are the following.

Neighborhood: A neighborhood of a graph $G$ in $X$ is the set $\{H \in X / \delta(H, G)<r\}, r \in \mathbf{R}^{+}$, and denoted by $V_{r}(G)$.

That is $V_{r}(G)=\{H \in X / \delta(H, G)<r\}$.

Open Set: A set $A \subset X$ is open if for each $G \in A$, exits $r \in \mathbf{R}^{+}$ such that $V_{r}(G) \subset A$.

Closed Set : A set $A \subset X$ is closed if $X-A$ is open.

Adherent Graph : A graph $G \in X$ is adherentgraph of set $A \subset X$ if for each $r \in \mathbf{R}^{+}, V_{r}(G) \cap A \neq \phi$. The set $\{G \in X / G$ is adherent graph an A\} is denoted by $\bar{A}$.

Remark 3.3: $. \bar{A}=\bigcap_{\substack{F \text { cerrado } \\ A \subset F}} \mathrm{~F}$

Theorem 3.4: $(X, \delta)$ is a metric complete space.

Proof: By proposition $3.2(X, \delta)$ is a metric space. Let $\left\{G_{i}\right\}_{i \in \mathbf{N}}$ be a sequence in $X$.

(i) Suppose that $\left\{G_{i}\right\}_{i \in \mathbf{N}}$ is a convergent sequence on graph $G$.

$\forall r \in \mathbf{R}^{+}, \exists \mathbf{n}_{\mathbf{r}} \in \mathbf{N}: \forall \mathbf{n} \in \mathbf{N}, n>n_{r}: G_{n} \in V_{\frac{r}{2}}(G) \Rightarrow$

$\Rightarrow \forall n, m \in \mathbf{N}, n, m>n_{r}: \delta\left(G_{n}, G_{m}\right) \leq \delta\left(G_{n}, G\right)+\delta\left(G, G_{m}\right)<$ $\frac{r}{2}+\frac{r}{2}=r \Rightarrow$

$\Rightarrow\left\{G_{i}\right\}_{i \in \mathbf{N}}$ is a Cauchy sequence.

(ii) Suppose that $\left\{G_{i}\right\}_{i \in \mathbf{N}}$ is a Cauchy sequence, i.e.,

$\forall r \in \mathbf{R}^{+}, \exists \mathbf{n}_{\mathbf{r}} \in \mathbf{N}: \forall \mathbf{n}, \mathbf{m} \in \mathbf{N}, n, m>n_{r}: \delta\left(G_{n}, G_{m}\right)<r$.

For each $n \in \mathbf{N}$, let $H_{n}=\overline{\bigcup_{p \in N} C\left(G_{n+p}\right)}$ be. According to lemma 3.1 each $C\left(G_{n+p}\right)$ is compact, then $H_{n}=\bigcup_{p \in N} C\left(G_{n+p}\right)$. As 
$H_{n}=C\left(G_{n}\right) \cup H_{n+1}$, then $H_{n+1} \subset H_{n}$. Let $G$ be a graph so that $C(G)=\bigcap_{n \in \mathbf{N}} H_{n}$. Since $\left\{H_{i}\right\}_{i \in \mathbf{N}}$ is decreasing, therefore $C(G) \neq \phi$ and accordingly $\lim _{n \rightarrow \infty} C\left(G_{n}\right)=C(G)$. For him previously affirmed, $\forall r \in \mathbf{R}^{+}, \exists \mathbf{n}_{\mathbf{r}} \in \mathbf{N}: \forall \mathbf{n} \in \mathbf{N}, n>n_{r}: \delta\left(G_{n}, G\right)<r$., and therefore $\left\{G_{i}\right\}_{i \in \mathbf{N}}$ is a convergent sequence on graph $\mathrm{G} \bullet$

Now, the following lemma is fundamental

Lemma 3.5: If $w$ is an application of $X$ in $X$, defined by $w(G)=$ $G(G)$, then $w$ is a contraction in $X$.

Proof: Choose $s \in\left[0,1\left[\right.\right.$ such that for each pair $G_{1}, G_{2} \in X$,

$\delta\left(w\left(G_{1}\right), w\left(G_{2}\right)\right) \leq s \delta\left(G_{1}, G_{2}\right)$. This $s$ number could be defined by means of an appropriate election of the metric in the realization of $G$ and $w(G)$ in $\mathbf{R}^{\mathbf{3}}$.

$$
\text { In fact } s=\operatorname{Sup}_{\substack{U, V \in X \\ U \neq V}} \frac{\delta(w(U), w(V))}{\delta(U, V)} \bullet
$$

The existence of an attractor point for $\mathrm{w}$ is assured by the following theorem whose demonstration could be found in [8].

Theorem 3.6: If $M$ is a metric complete space and $f: M \rightarrow M$ is a contraction, then $x_{f}=n \rightarrow \infty \lim f^{n}(x)$ it exist and it is independent of the election of $x$ in $M$. Also, $x_{f}$ is the only fixed point of $f$.

Theorem 3.7: If $G \in X$, then the orbit $G \rightarrow w(G) \rightarrow \ldots \rightarrow$ $w^{k}(G) \rightarrow$...has a single attractor point $G_{w}$ for $w$.

Proof: Applying lemma 3.5 and theorem 3.6.

3. The algorithm[4]. Observe that the described previously is of deterministic character, due to this fact an deterministic algorithm in order to calculate the attractor point of w. An analysis shows that if $G$ is a graph with $p$ vertices then $w(G)$ has $p^{2}$ vertices and, in general, $w^{k}(G)$ has $p^{k}$ vertices. The algorithm to be used generates the $G$ graph, after $w(G)$ and so on. Figure 4 shows an example of an orbit of $w$ for $G=K_{5}$. 

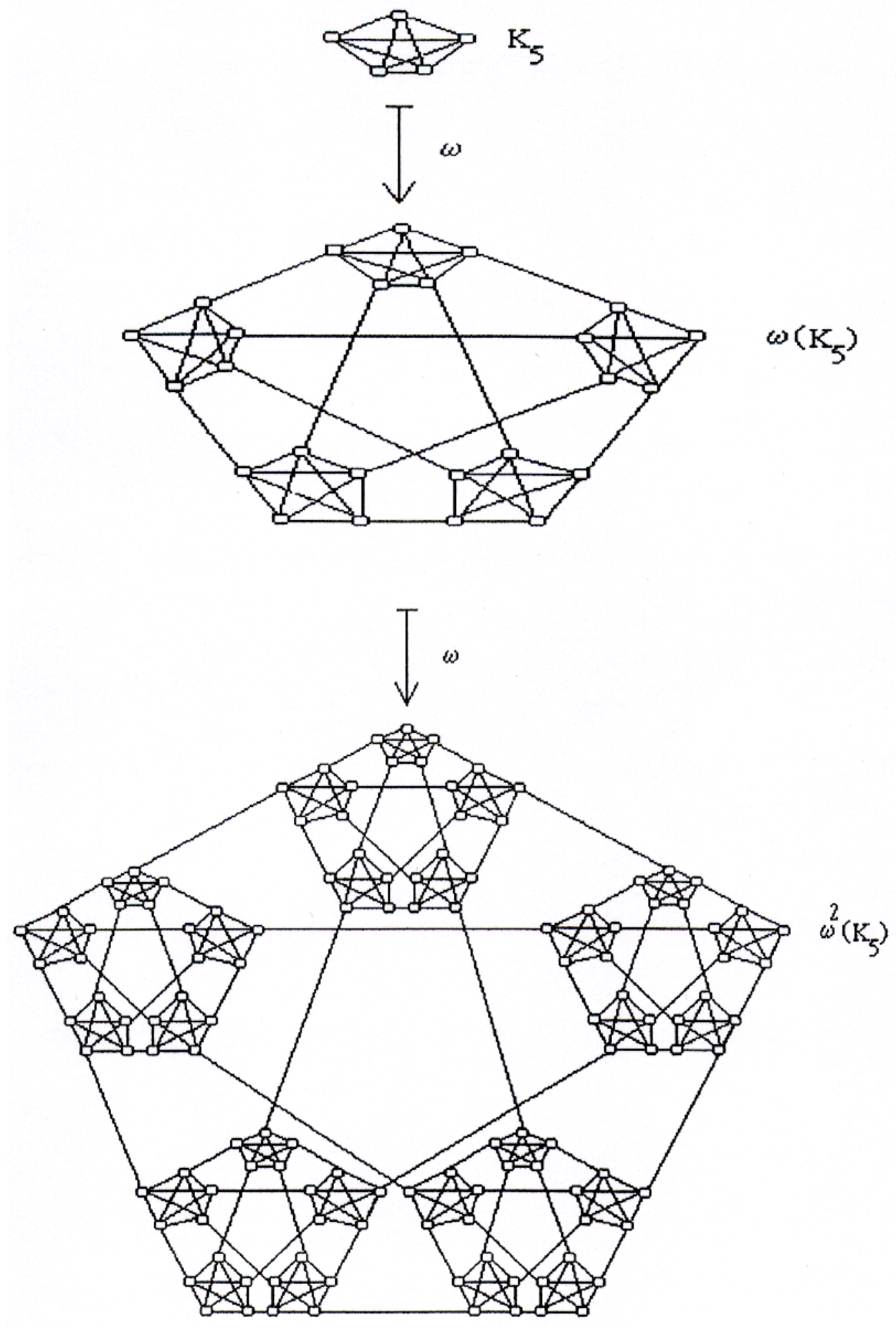

FIGURE 4 


\section{References}

[1] M. BARNSLEY, Fractal Everywhere, Academic Press, (1988).

[2] A. BRONDSTED, An Introduction to Convex Polytopes, Springer Verlag, New York, Heidelberg, Berlin, (1983).

[3] G. CHARTRAND, LESNIAIK, L., Graphs and Digraphs, Wadsworth and Brooks/Cole Advanced Books and Software Pacific Grove, C. A., (1996).

[4] G. CHARTRAND AND O. OELlERMANN, Applied and Algorithmic Graph Theory, McGraw-Hill., Inc., (1993).

[5] H. COXETER, Regular Polytopes, Third Edition, Dover Publication, Inc, (1973).

[6] R. DEVANEY, Introduction to Chaotic Dynamical Systems, 2nd edition, Addison-Wesley, (1989).

[7] R. HOLMGREN, A First Course in Discrete Dynamical Systems, Springer-Verlag, (1994).

[8] A.N. KOLMOGOROV \& S.V.FOMIN, Introductory Real Analysis, Dover Publications, INC., New York, (1975).

[9] E. MONTENEGRO, R. SALAZAR, A result about the incidents edges in the graphs $M_{k}$, Discrete Mathematics, 122, pp. 277-280, (1993).

[10] E. MONTENEGRO, D. POWERS, S. RUIZ, R. SALAZAR, Spectra of related graphs and Self Reproducing Polyhedra, Proyecciones, 11, $\mathrm{N}^{\circ} 1$, pp. 01-09, (1992)

[11] E. MONTENEGRO, D. POWERS, S. RUIZ, R. SALAZAR, Automorphism Group and hamiltonian properties preserved by Substitution, Scientia, Serie A Math. Sciences 4, pp. 57-67, (1993). 
[12] E. PRISNER, Graph Dynamics, version 2B, Universität Hamburg, Hamburg, F.R. Germany, (1994).

[13] R. ROCKAFELLAR, Convex Analysis, Princeton University Press, (1970).

Received : May, 2000

\section{Eduardo Montenegro}

Instituto de Matemáticas

Universidad Católica de Valparaíso

Blanco Viel N 596

Cerro Baron

Valparaíso

Chile

e-mail : emontenv@ucv.cl

and

\section{Eduardo Cabrera}

Facultad de Ciencias Naturales y Exactas

Universidad de Playa Ancha

Casilla 34-V

Valparaíso

Chile

e-mail : emontene@upa.cl 